\title{
Pilot Decontamination over Time Frequency and Space Domains in Multi-Cell Massive MIMO System
}

\author{
Lamiae Hachad, Fatiha Mrabti, Hicham Ghennioui, Mohcine Zouak
}

Sidi Mohamed Ben Abdellah University, Faculty of Sciences and Technologies Fez, LSSC, Morocco

\begin{tabular}{l}
\hline \hline Article Info \\
\hline Article history: \\
Received Jan 18, 2018 \\
Revised Apr 23, 2018 \\
Accepted Apr 30, 2018 \\
\hline Keyword: \\
5G \\
El-Az estimation \\
Massive MIMO \\
Pilot contamination \\
Time-frequency analysis
\end{tabular}

\begin{abstract}
In this article, we show that Pilot contamination problem can be seen as a source separation problem using time, frequency, and space domains. Our method capitalizes on a nonunitary joint diagonalization of spatial quadratic time-frequency (STFD) signal representation to identify the desired and interfering users. We first compute the noise subspace from the STFD matrices selected appropriately. Secondly, we use the noise subspace obtained to estimate the Elevation (El) and the Azimuth (Az) angles for which the MUSIC cost function is maximized. Numerical simulations are provided to illustrate the effectiveness and the behavior of the proposed approach.
\end{abstract}

Copyright $\odot 2018$ Institute of Advanced Engineering and Science. All rights reserved.

\section{Corresponding Author:}

Lamiae Hachad,

Faculty of Sciences and Technologies Fez, LSSC,

Sidi Mohamed Ben Abdellah University,

Route Immouzzer, B.P. 2202, Fez, Morocco.

Email: lamiae.hachad@usmba.ac.ma

\section{INTRODUCTION}

Increased capacity demands, improved throughput, and additional use cases for wireless access where all things will be connected in a seamless fashion have steered a new vision of mobile broadband communications [1]-[4]. The 5th generation (5G) entails new technologies, which have to fulfill many requirements such as the high data rates per user, uniformly good service throughout the coverage area and the ability to function under high mobility conditions, etc. The deployment of small cells and exploitation of higher frequencies are known as 5G key technologies, nevertheless they are unable alone to address these needs.

Massive MIMO (Multi Input and Multi Output) is the new and the most promising direction in mobile access that paves the way for 5G [5]. This emerging technique is an eventual and worthwhile form of the Multi User MIMO called also Large scale antenna system, very large MIMO, or hyper MIMO [6]-[14]. It scales up conventional MIMO by possibly of degrees of magnitude compared to the current state-of-the-art. The Massive MIMO technique could constitute a network of cells, or it could be an isolated (single-cell) site where antenna arrays with a very large number of physically small, non-directive antennas are employed to serve a multiplicity of autonomous users (terminals with a single antenna) in the same time-frequency resources.

Generally, Massive MIMO Systems operate in Time Division Duplex (TDD) mode and rely on reciprocity between the uplink and downlink channels. It is an obvious choice for two good reasons. Firstly, the information about the channels is required only by the BS to process the antennas coherently. Secondly, the uplink estimation overhead is proportional to the number of terminals but independent of service antennas number. However, the uplink resources required in the Frequency Division Duplex mode (FDD) to inform 
the BS about the channel responses would be drastically bigger and proportional to the number of antennas at the base station [15]. In spite of that fact, the FDD operation may be possible in certain cases [16].

On TDD massive MIMO systems, an orthogonal uplink pilot sequence is assigned to every terminal. In fact, the limited duration of the coherence interval is the main reason that the orthogonal pilot sequences are normally reused by users in neighboring cells. In [17], for a typical operating scenario, the maximum number of orthogonal pilot sequences, in a one-millisecond coherence interval, is estimated to be about 200. The accessible supply of orthogonal pilot sequences in a multi-cellular system is typically expended. Hence, the necessity of reusing non-fully orthogonal pilot sequences across all the cells. Unfortunately, the multi-cell massive MIMO system would be plagued by the so-called pilot contamination. In particular, a linear combination of terminals' channels having the same pilot sequence contaminates the estimated channel obtained in the service array.

The pilot contamination has a detrimental impact on the actual achievable spectral and energy efficiencies in real systems [18]-[20]. As a result, considerable research efforts have been spent in the last years towards alleviating pilot interference in massive MIMO networks. It has been demonstrated in [21] that the effect of pilot contamination can be exterminated using a Bayesian channel estimation ensuring certain conditions of the channel covariance. The main idea in [21] is to assign the same pilot to users revealing maximum orthogonality of signal subspaces (with the same Direction Of Arrival DOA). Besides, all covariance matrices of all channels must be learned previously which incurs too much coordination. Another nonlinear channel estimation technique is proposed in [22] as a blind pilot decontamination method. It consists of exploiting the difference between the channel gains of the desired users and the interfering users in order to discriminate users in the amplitude domain. This approach was considered in [23] for a more sensible physical channel model. The authors discussed the ability of subspace method to identify the desired and interfering users. It was proven that the subspace approach works well within the constraint that the interference channel is received with a power level sufficiently lower than that of the desired channel.

In effect, most of the previous works considered the Elevation parameter and/or the constraint of a larger power ratio between the intended users and the interfering ones. Nonetheless, in real channels, the Elevation parameter (1D DOA) is a finite number and can be identic in some scenarios such a rich scattering environment. As well, the constraint of power ratio is a condition hard to guarantee for some edge-of-cell users as they may receive their signals with the same Elevation angle (El) and with the same power.

Therefore, we aim in this work to propose an effective solution to the pilot contamination problem for all cell users and more precisely taking into account those edge-of-cell users. Our idea behind is to state and resolve the pilot contamination problem as a source separation problem using time, frequency, and space domains. The mixing matrix compromises both the interfering and desired sources propagation channels. The latter is defined by the sources spatial signatures bearing angle that will be recovered by a DOA estimation technique. Assuming that 1D DOA does not allow separating the intended users and the interfering ones since the signals of two users may be received with the same direction, we propose to include both $\mathrm{El}$ and $\mathrm{Az}$ angles to separate the users of each cell. We assume that the probability of two users having the same values for both elevation and azimuth angles is very small.

In this paper, we exploit the concept of STFD [24]-[26]. It has been used to provide high resolution and enable separations of signals corrupted by interference occupying the same frequency band and/or the same time segment. It offers an improving signal selectivity over approaches using the covariance matrix. The correlation matrix is a low-dimension representation of STFD, the reason that STFD-based methods offer better performance than conventional covariance matrix based approaches. Afterward, we apply a nonunitary Joint diagonalization (JD) method to a combined set of Spatio-temporal correlation matrices, which is selected using a Time-Frequency TF point detector cited in [26] to provide an improved estimate of the signal and noise subspaces over the standard Singular Value Decomposition (SVD) technique. Finally, the Multiple Signal Classification (MUSIC) technique is used to determine the values of El and Az angles. The result is an improved sources separation using the parameters: time, frequency, and space variables.

The outline of this paper is as follows. We describe in Section 2 the problem formulation and derive the concept of the STFD representations for a Multi-Cell Massive MIMO system. We discuss the challenges for selecting TF points and present the method applied for selecting the TF points. We introduce also the NUJD approach used for estimated the noise subspace that is used in its turn to determine the azimuth and elevation angles using MUSIC spectrum. Simulations results and conclusion are presented in Sections 3 and Section 4.

Int J Elec \& Comp Eng, Vol. 8, No. 5, October 2018 : 3609 - 3619 


\section{PROBLEM FORMULATION}

\subsection{System model}

We will consider the uplink in multi-cell multi-user MIMO communication system with L cells as shown in Figure 1, with full spectrum reuse. There are $\mathrm{K}$ single-antenna users in each cell simultaneously served by their Base Station (BS) equipped with a Uniform Rectangular Array (URA) as shown in Figure 2. The cellular network operates in time-division duplexing (TDD) mode and the channel is assumed to be a block-fading channel, which remains constant over a coherence time of $\mathrm{C}$ symbol periods, and changes independently from one coherence time to another. The same pilot pool is reused among all cells and the pilot sequences are assumed orthogonal to each other in order to avoid intra-cell interference.

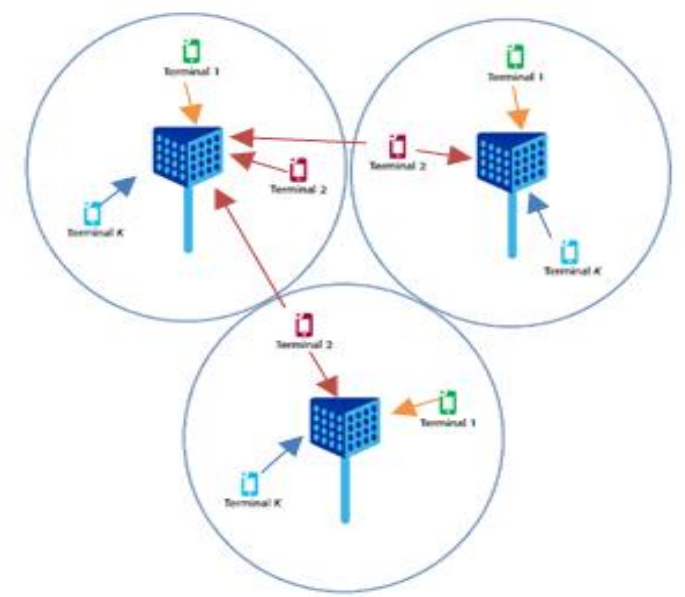

Figure 1. The concept of pilot contamination in a multi cell massive MIMO system

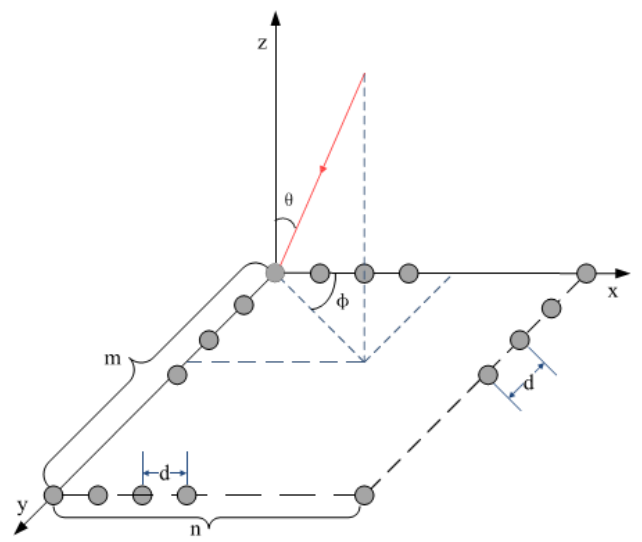

Figure 2. System model of $\mathrm{M} \times \mathrm{N}$ URA configuration

In this paper, we consider that the carrier frequency is $3 \mathrm{GHz}$ and the URA is selected with the consideration of limit space. The antenna array at the base station is placed in the X-Y plane with $\mathrm{M}$ antenna elements in each column and $\mathrm{N}$ antenna elements in each row. The spacing between adjacent antenna elements is assumed to be half wavelength $(\mathrm{d} \leq \lambda / 2)$ in both two dimensions. The DOA is depicted by elevation and azimuth angles, given by $\theta \in\left\{0^{\circ}, 90^{\circ}\right\}$ and $\phi \in\left\{-90,{ }^{\circ} 90^{\circ}\right\}$ respectively [27], [28].

The so-called steering vector quantifies the array response in a given direction. Hence, the channel from the $\mathrm{j}^{\text {th }}$ user in the $\mathrm{i}^{\text {th }}$ cell to the intended BS is denoted by $\mathrm{h}_{\mathrm{ji}}$. We adopt the Correlation Channel Model [29] given by:

$$
\mathbf{h}_{\mathrm{ji}}=\alpha_{\mathrm{ji}} \mathbf{a}_{\mathrm{ji}}\left(\theta_{\mathrm{ji}}, \phi_{\mathrm{ji}}\right)
$$


Where

a. aji : is the steering vector corresponding to the angles of arrival, the elevation $\theta_{\mathrm{ji}}$ and the azimuth $\phi_{\mathrm{ji}}$ associated with the $\mathrm{j}^{\text {th }}$ user.

b. $\alpha_{\mathrm{ji}} \sim \mathcal{C} \mathcal{N}\left(0, \beta_{\mathrm{ji}}\right)$ : is the channel gain.

Moreover, it is assumed that all $\theta_{\mathrm{ji}}, \phi_{\mathrm{ji}}$ et $\alpha_{\mathrm{ji}}$ are independent over user index $\mathrm{j}$ and cell index $\mathrm{i}$. The $\mathrm{MN} \times 1$ manifold $\mathbf{a}_{\mathbf{j i}}\left(\theta_{\mathrm{ji}}, \phi_{\mathrm{ji}}\right)$ is given by

$$
\begin{aligned}
& \mathbf{a}_{\mathrm{ji}}\left(\theta_{\mathrm{ji}}, \phi_{\mathrm{ji}}\right)=\operatorname{vec}\left\{\left[1, \mathrm{e}^{\frac{2 \pi \mathrm{d}}{\lambda} \sin \left(\theta_{\mathrm{ji}}\right)}, \ldots, \mathrm{e}^{\frac{2 \pi \mathrm{d}}{\lambda}(\mathrm{N}-1) \sin \left(\theta_{\mathrm{ji}}\right)}\right]^{\mathbf{T}}\right. \\
& \left.\otimes\left[1, \mathrm{e}^{\frac{2 \pi \mathrm{d}}{\lambda} \sin \left(\phi_{\mathrm{ji}}\right)}, \ldots, \mathrm{e}^{\frac{2 \pi \mathrm{d}}{\lambda}(\mathrm{M}-1) \sin \left(\phi_{\mathrm{ji}}\right)}\right]^{\mathrm{T}}\right\}
\end{aligned}
$$

Here after $\otimes$ et $\operatorname{vec}\{$.$\} operators the Kronecker product and the vectorized operation respectively.$

Let $\mathbf{H}_{\mathrm{i}} \triangleq\left(\mathbf{A}_{1 i} \tilde{\mathrm{h}}_{1 i}, \ldots, \mathbf{A}_{K i} \tilde{\mathrm{h}}_{K i}\right)$ be the $\mathrm{MN} \times \mathrm{K}$ effective fast-fading channel from $\mathrm{K}$ users in the $i^{\text {th }}$ cell to the intended $\mathrm{BS}$, where:

a. $\mathbf{A}_{j i}$ is the steering vector of the $j^{\text {th }}$ user in the $i^{\text {th }}$ cell;

b. $\tilde{\mathrm{h}}_{j i} \in \mathbb{C}^{K \times 1}$ consists of i.i.d $\mathcal{C N}(0,1)$.

The $\mathrm{MN} \times \mathrm{C}$ signal received by the 1 -th $\mathrm{BS}$ during $\mathrm{C}$ consecutive symbol intervals may be written as :

$$
\boldsymbol{Y}=\mathbf{H}_{1} \mathbf{X}_{1}+\sum_{\mathrm{i}=2}^{\mathrm{L}} \mathbf{H}_{\mathrm{i}} \mathbf{X}_{\mathrm{i}}+\mathbf{B}
$$

where $\mathbf{B}$ is an additive noise vector whose entries are considered generally as stationary, temporally and spatially white, zero mean random processes, and independent of the sources signals.

The Equation (3) can be simplified to the following equation:

$$
\mathbf{Y}=\mathbf{H X}+\mathbf{Z}
$$

$\mathbf{X}=\mathbf{X}_{1} \in \mathbb{C}^{\mathrm{K} \times \mathrm{C}}$ is the transmitted signals from the intended cell sources through the channel $\mathbf{H}=\mathbf{H}_{1} \in \mathbb{C}^{\mathrm{MN} \times \mathrm{K}}$ and the impairment process $\mathbf{Z} \in \mathbb{C}^{\mathrm{MN} \times \mathrm{C}}$ is decomposed into white noise $\mathbf{B}$ and interference from $\mathrm{L}-1$ neighboring cells

$$
\mathbf{Z}=\mathbf{H}_{\mathrm{I}} \mathbf{X}_{\mathbf{I}}+\mathbf{B}
$$

where the data $\mathbf{X}_{\mathrm{I}} \in \mathbb{C}^{(\mathrm{L}-1) \mathrm{K} \times \mathrm{C}}$ is transmitted from interfering cells and received in the cell of interest through the channel $\mathbf{H}_{\mathrm{I}} \in \mathbb{C}^{\mathrm{MN} \times(\mathrm{L}-1) \mathrm{K}}$.

Now, we consider the nonlinear model of the studied system defined in Equations (4) and (5), the STFD of the dimension MN $\times$ MN is denoted as

$$
\begin{aligned}
& \mathbf{D}_{\mathbf{Y Y}}(\mathrm{t}, v)=\mathbf{H D}_{\mathbf{X X}}(\mathrm{t}, v) \mathbf{H}^{\mathrm{H}}+\mathbf{H D}_{\mathbf{X X}_{\mathbf{I}}}(\mathrm{t}, \mathrm{v}) \mathbf{H}_{\mathbf{I}}^{\mathbf{H}}+\mathbf{H}_{\mathbf{I}} \mathbf{D}_{\mathbf{X}_{\mathbf{I}} \mathbf{X}}(\mathrm{t}, \mathrm{v}) \mathbf{H}^{\mathbf{H}}+\mathbf{H}_{\mathbf{I}} \mathbf{D}_{\mathbf{X}_{\mathbf{I}} \mathbf{X}_{\mathbf{I}}}(\mathrm{t}, \mathrm{v}) \mathbf{H}_{\mathbf{I}}^{\mathbf{H}}+\mathbf{H D}_{\mathbf{X B}}(\mathrm{t}, \mathrm{v})+ \\
& \mathbf{D}_{B X}(t, v) \mathbf{H}^{H}+\mathbf{H}_{I} \mathbf{D}_{\mathbf{X}_{I} B}(\mathrm{t}, \mathrm{v})+\mathbf{D}_{\mathbf{B X}_{I}}(\mathrm{t}, \mathrm{v}) \mathbf{H}_{\mathrm{I}}^{\mathrm{H}}+\mathbf{D}_{\mathrm{BB}}(\mathrm{t}, \mathrm{v})
\end{aligned}
$$

where:

a. $\mathbf{D}_{\mathbf{X X}}(t, v)$ represents the STFD of the intended cell sources,

b. $\mathbf{D}_{\mathbf{X}_{\mathbf{I}} \mathbf{X}_{\mathbf{I}}}(\mathrm{t}, \mathrm{v})$ is the STFD of the interference

c. $\mathbf{D}_{\mathbf{X X}_{\mathbf{I}}}(\mathrm{t}, \mathrm{v})$ and $\mathbf{D}_{\mathbf{X}_{\mathbf{I}} \mathbf{X}}(\mathrm{t}, \mathrm{v})$ are the crossed STFDs between the sources and interference from $\mathrm{L}-1$ neighboring cells

d. $\mathbf{D}_{\mathbf{X B}}(\mathrm{t}, \mathrm{v})$ and $\mathbf{D}_{\mathbf{B X}}(\mathrm{t}, \mathrm{v})$ symbolize the crossed STFDs between the intended cell sources and noise,

e. $\mathbf{D}_{\mathbf{X}_{\mathbf{I}} \mathbf{B}}(t, v)$ and $\mathbf{D}_{\mathbf{B X}_{\mathbf{I}}}(t, v)$ are the crossed STFDs between the interference from $L-1$ neighboring cells and noise.

f. $\mathbf{D}_{\mathrm{NN}}(\mathrm{t}, \mathrm{v})$ represents the STFD of the Noise. 
Under the assumption of white noise, the expectation of the STFD matrices between the intended sources signals and the noise $\mathbf{D}_{\mathbf{X B}}(\mathrm{t}, \mathrm{v})$, and $\mathbf{D}_{\mathbf{B X}}(\mathrm{t}, \mathrm{v})$ and between the interference from L neighboring cells and noise $\mathbf{D}_{\mathbf{X}_{\mathbf{I}} \mathbf{B}}(\mathrm{t}, \mathrm{v})$ and $\mathbf{D}_{\mathbf{B X}_{\mathbf{I}}}(\mathrm{t}, \mathrm{v})$ vanish, i.e.

$$
\begin{aligned}
& \mathrm{E}\left[\mathbf{D}_{\mathbf{X B}}(t, v)\right]=0, E\left[\mathbf{D}_{\mathbf{B X}}(t, v)\right]=0, \\
& \mathrm{E}\left[\mathbf{D}_{\mathbf{X}_{\mathbf{I}} \mathbf{B}}(t, v)\right]=0, E\left[\mathbf{D}_{\mathbf{B X}_{\mathbf{I}}}(t, v)\right]=0
\end{aligned}
$$

The interference is not independent of the data X, hence the STFD of the observations becomes:

$$
\begin{aligned}
& \boldsymbol{D}_{Y \boldsymbol{Y}}(t, v)=\boldsymbol{H} \boldsymbol{D}_{\boldsymbol{X} \boldsymbol{X}}(t, v) \boldsymbol{H}^{H}+\boldsymbol{H} \boldsymbol{D}_{\boldsymbol{X} \boldsymbol{X}_{\boldsymbol{I}}}(t, v) \boldsymbol{H}_{\boldsymbol{I}}^{\boldsymbol{H}}+\boldsymbol{H}_{\boldsymbol{I}} \boldsymbol{D}_{\boldsymbol{X}_{\boldsymbol{I}} \boldsymbol{X}}(t, v) \boldsymbol{H}^{\boldsymbol{H}}+\boldsymbol{H}_{\boldsymbol{I}} \boldsymbol{D}_{\boldsymbol{X}_{\boldsymbol{I}} \boldsymbol{X}_{\boldsymbol{I}}}(t, v) \boldsymbol{H}_{\boldsymbol{I}}^{\boldsymbol{H}}+ \\
& \boldsymbol{D}_{\boldsymbol{B} \boldsymbol{B}}(t, v)
\end{aligned}
$$

\subsection{Pilot decontamination using time-frequency El-Az estimation}

The proposed method for pilot contamination problem can be summarized in Figure 3 follows

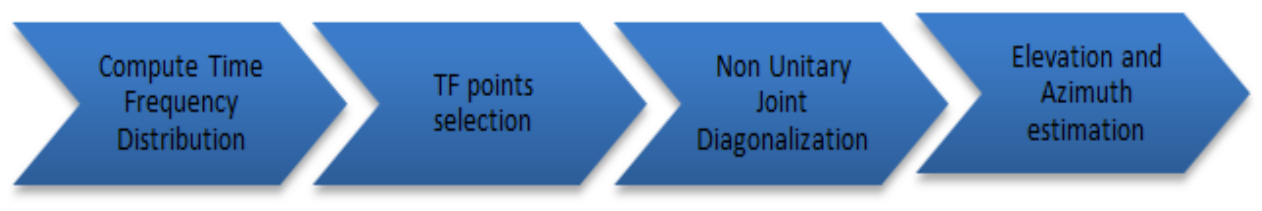

Figure 3. The Organogram of the proposed method NU-TF-MUSIC El-Az estimation

El-Az estimation can only be materialized if appropriate TF points are selected in the STFD matrices formulation as shown in Equation (7).

\subsubsection{Time-frequency points selection}

We use the same TF points detector proposed for the instant context. It concerns finding useful TF points through inspecting the time frequency points where the STFD observations matrices are rank one. For this reason, a Singular Value Decomposition (SVD) is used leading to

$$
\mathbf{D}_{\mathbf{Y Y}}(\mathrm{t}, \mathrm{v})=\left(\mathbf{U}(\mathrm{t}, \mathrm{v}) \boldsymbol{\Delta}(\mathrm{t}, \mathrm{v}) \mathbf{V}(\mathrm{t}, \mathrm{v})^{\mathrm{H}}\right)
$$

with $\mathbf{V}(\mathrm{t}, \mathrm{v})$ et $\mathbf{U}(\mathrm{t}, \mathrm{v})$ are two $M N \times M N$ unitary matrices, and $\boldsymbol{\Delta}(\mathrm{t}, \mathrm{v})=\operatorname{diag}\{\delta(\mathrm{t}, \mathrm{v})\}$ is a diagonal matrix whose elements are positive. Let $\delta(\mathrm{t}, \mathrm{v})=\left(\delta_{1}(\mathrm{t}, \mathrm{v}), \ldots, \delta_{K}(\mathrm{t}, \mathrm{v})\right)^{T}$ and nd assume that the singular values are arranged in a decreasing order $\delta_{1}(\mathrm{t}, \mathrm{v}) \geq \delta_{2}(\mathrm{t}, \mathrm{v}) \geq \cdots \geq \delta_{K}(\mathrm{t}, \mathrm{v}) \geq 0$. Therefore, we choose a point $(\mathrm{t}, \mathrm{v})$ and check if a matrix is rank one according to the following two conditions.

$$
\left\{\begin{array}{c}
\boldsymbol{\delta}_{1}(\mathrm{t}, \mathrm{v})>\varepsilon_{1} \\
\sum_{\mathrm{i}=2}^{\mathrm{M}} \boldsymbol{\delta}_{\mathrm{i}}(\mathrm{t}, \mathrm{v})<\varepsilon_{2}
\end{array}\right.
$$

where $\varepsilon_{1}$ (resp. $\left.\varepsilon_{2}\right)$ is a positive constant sufficiently large (resp. small). The set of $\mathrm{N}_{M}\left(\right.$ with $\left.\mathrm{N}_{M} \in \mathrm{N}^{*}\right)$ of matrices $\mathcal{M}$ which is being built, all admit a particular structure such that

$$
\mathbf{D}_{\mathbf{Y Y}}(\mathrm{t}, \mathrm{v})=\mathbf{H} \mathbf{D}_{\mathbf{X X}}(\mathrm{t}, \mathrm{v}) \mathbf{H}^{\mathrm{H}}+\mathbf{H D}_{\mathbf{X X}}(\mathrm{t}, \mathrm{v}) \mathbf{H}_{\mathbf{I}}^{\mathbf{H}}+\mathbf{H}_{\mathbf{I}} \mathbf{D}_{\mathbf{X}_{I} \mathbf{X}}(\mathrm{t}, \mathrm{v}) \mathbf{H}^{\mathbf{H}}+\mathbf{H}_{\mathbf{I}} \mathbf{D}_{\mathbf{X}_{I} \mathbf{X}_{\mathbf{I}}}(\mathrm{t}, \mathrm{v}) \mathbf{H}_{\mathbf{I}}^{\mathbf{H}}
$$

with $\mathbf{D}_{\mathbf{X X}}(\mathrm{t}, \mathrm{v}), \mathbf{D}_{\mathbf{X X}}(\mathrm{t}, \mathrm{v}), \mathbf{D}_{\mathbf{X}_{\mathbf{I}} \mathbf{X}}(\mathrm{t}, \mathrm{v})$ and $\mathbf{D}_{\mathbf{X}_{\mathbf{I}} \mathbf{X}_{\mathbf{I}}}(\mathrm{t}, \mathrm{v})$ are diagonal matrices with a single non-zero term on the diagonal.

\subsubsection{Joint diagonalization}

We consider the set $\mathcal{M}$ built using the detector that has just been presented in the previous section.

$$
\boldsymbol{M}=\left\{\mathrm{M}_{\mathrm{i}}=\mathbf{D}_{\mathrm{YY}}\left(\mathrm{t}_{\mathrm{i}}, v_{\mathrm{i}}\right) \in \mathbb{C}^{\mathrm{M} \times \mathrm{M}}, \forall \mathrm{i}=1, \ldots, \mathrm{N}_{\mathrm{M}}\right\}
$$


All matrices of the set $\mathcal{M}$ admit the following decomposition:

$$
\mathbf{M}_{\mathbf{i}}=\mathbf{E} \mathbf{D}_{\mathbf{i}} \mathbf{E}^{\mathrm{H}} \text { or } \mathbf{D}_{\mathbf{i}}=\mathbf{F M}_{\mathbf{i}} \mathbf{F}^{\mathrm{H}} \forall \mathrm{i} \in\left\{1, \ldots, \mathrm{N}_{\mathrm{M}}\right\}
$$

where:

$$
\mathbf{D}_{\mathbf{i}}=\left(\begin{array}{cccc}
\mathrm{d}_{\mathrm{i}, 11} & 0 & \cdots & 0 \\
0 & \mathrm{~d}_{\mathrm{i}, 22} & \ddots & \vdots \\
\vdots & \ddots & \ddots & 0 \\
0 & \cdots & 0 & \mathrm{~d}_{\mathrm{i}, \mathrm{LKLK}}
\end{array}\right) \forall \mathrm{i}\left\{1, \ldots, \mathrm{N}_{\mathrm{M}}\right\}
$$

Are diagonal matrices of dimension $\operatorname{LK} \times \operatorname{LK}$ and $\mathrm{d}_{\mathrm{i}, \mathrm{j}, \mathrm{k}}, \mathrm{i} \in\left\{1, \ldots, \mathrm{N}_{\mathrm{M}}\right\}, \mathrm{j}=\mathrm{k} \in\{1, \ldots, \mathrm{LK}\}$ are the autoterms. The matrix $\mathbf{E}$ is a full rank matrix of dimension $M N \times L K$ and $\mathbf{F}$ is its pseudo-inverse matrix.

The problem of nonunitary joint diagonalization is to estimate the separation matrix $\widehat{\mathbf{A}}$ only from the matrices of the set $\boldsymbol{M}$. We consider the following criterion:

$$
\mathrm{C}_{\mathrm{JD}}(\widehat{\mathbf{A}})=\sum_{\mathrm{i}=1}^{\mathrm{N}_{\mathrm{M}}}\left\|\operatorname{Off} \operatorname{diag}\left\{\widehat{\mathbf{A}} \mathbf{M}_{\mathbf{i}} \widehat{\mathbf{A}}^{\mathrm{H}}\right\}\right\|_{\mathrm{F}}^{2}
$$

where $\|.\|_{F}$ denotes the Frobenius norm and the $\mathbf{O f f}$ diag\{.\}operator extracts the diagonal elements of a matrix to build a zero diagonal matrix. In order to find the estimated $\widehat{\mathbf{A}}$, an estimate of the steering matrix $\mathbf{A}$, we need to minimize the sum of squared norms non-diagonal elements of $\widehat{\mathbf{A}} \mathbf{M}_{\mathbf{i}} \widehat{\mathbf{A}}^{\mathrm{H}}$. This is done as in [29] through an algebraic solution based on computing the exact Hessian complex matrices.

\subsubsection{El-Az estimation}

The El-Az estimation is accomplished using NU-TF-MUSIC, which looks for peaks in a spatial spectrum computed for every pair of angle in a region of interest. To describe NU-TF-MUSIC, we denote $\widehat{\mathbf{E}}_{\mathrm{z}}$ as the noise subspace of the estimated steering matrix obtained by jointly diagonalizing the STFD matrix. The estimation technique MUSIC estimates jointly the $\mathrm{El}$ and $\mathrm{Az}$ angles by determining the LK values of $\theta$ and $\phi$ for which the following spatial spectrum is maximized,

$$
\mathrm{f}_{\text {MUSIC }}^{\mathrm{TF}}(\theta, \phi)=\frac{1}{\left[\mathbf{a}(\theta, \phi)^{\mathrm{H}} \hat{\mathbf{E}}_{\mathbf{z}} \hat{\mathbf{E}}_{\mathbf{Z}}^{\mathrm{H}} \mathbf{a}(\theta, \phi)\right]^{\mathrm{T}}}
$$

Once the noise subspace is obtained, the $\mathrm{El}$ and $\mathrm{Az}$ angles are determined by locating the LK peaks of the spatial spectrum. After all, the pilot contamination problem is cast as sources separation problem as we obtained the El and Az angles of every distinguished user. The BS can establish its users' channels and edgeof-cell users' channels without falling back on the constraint of power control to ensure that the intended users' power is upper than the interfering users power. Consequently, the effect of pilot contamination is eliminated.

\section{PERFORMANCE EVALUATION}

In this section, we evaluate the performance of the proposed approach in a multi-Cell Massive MIMO context by conducting Monte Carlo simulations. In the simulation, we consider two hexagonally shaped adjacent cells in the network. Each base station has a total number of 128 antenna elements, which is considered as one possible dimension of massive MIMO [30]. The 128 antenna elements are extended over a $\mathrm{M} \times \mathrm{N}$ dimension forming a URA configuration, with half wavelength antenna spacing. There are four users per cell using the same pilot sequences and having the same elevation angle values where $\theta_{\text {cell } 1}=\left\{5^{\circ}, 15^{\circ}, 25^{\circ}, 35^{\circ}\right\} \theta_{\text {cell } 2}=\left\{5^{\circ}, 15^{\circ}, 25^{\circ}, 35^{\circ}\right\}, \quad \phi_{\text {cell } 1}=\left\{0^{\circ}, 8^{\circ}, 20^{\circ}, 90^{\circ}\right\} \quad$ and $\phi_{\text {cell } 2}=\left\{10^{\circ}, 30^{\circ}, 40^{\circ}, 60^{\circ}\right\}$. We consider the root-mean-squared error (RMSE) as a performance metric to measure the effectiveness of El-Az estimation and sources separation, which is defined as:

$$
\operatorname{RMSE}=\sqrt{\frac{1}{\mathrm{LK}} \frac{1}{\mathrm{~N}_{\mathrm{mc}}} \sum_{\mathrm{k}=1}^{\mathrm{LK}} \sum_{\mathrm{n}=1}^{\mathrm{N}_{\mathrm{mc}}}\left[\left(\hat{\theta}_{\mathrm{k}}-\theta_{\mathrm{k}}\right)^{2}+\left(\widehat{\phi}_{\mathrm{k}}-\phi_{\mathrm{k}}\right)^{2}\right]}
$$

where $\widehat{\theta}_{\mathrm{k}}$ and $\widehat{\phi}_{\mathrm{k}}$ are the estimation of elevation angle $\theta_{\mathrm{k}}$ and the estimation of azimuth angle $\phi_{\mathrm{k}}$ respectively at the $\mathrm{n}^{\text {th }}$ Monte Carlo trial. LK is the estimated source number and $\mathrm{N}_{\mathrm{mc}}$ represents Monte Carlo simulation 
times. We first show the spectrum of the proposed method, NU-TF-MUSIC, based only on the elevation angle for eight sources, where the four interfering sources and the four intended sources have the same values. The simulation is conducted with a coherence interval of $\mathrm{C}=128$, and a value of $\mathrm{SNR}=15 \mathrm{~dB}$.

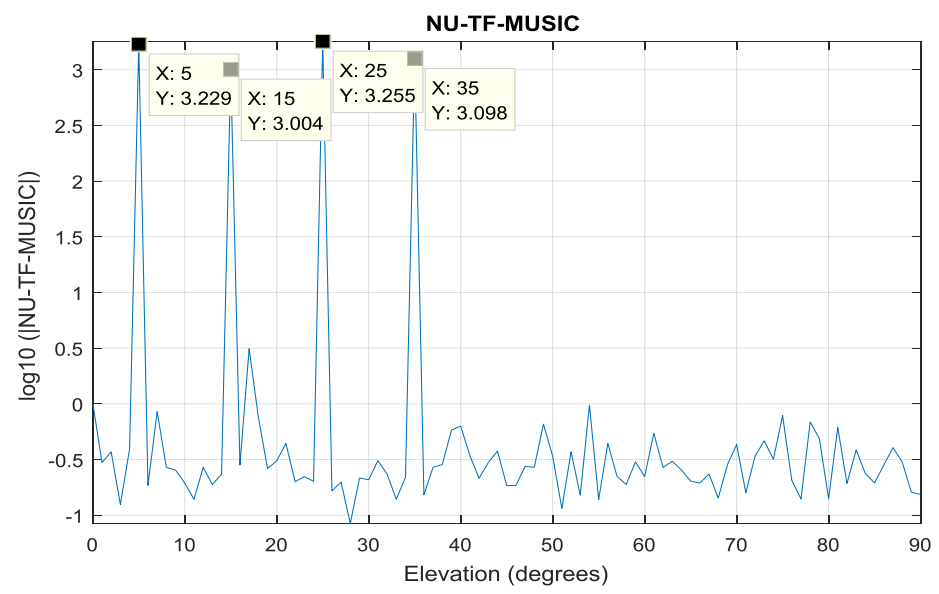

Figure 1. NU-TF-MUSIC spectrum for a SNR value of $15 \mathrm{~dB}$

As we can observe in Figure 1, the spectrum exhibits just four peaks. One peak for each elevation angle $\left(\left\{5^{\circ}, 15^{\circ}, 25^{\circ}, 35^{\circ}\right\}\right)$. We cannot decide which user location is displayed in the figure. We can conclude that 1D DOA (only the elevation) does not allow discriminating users of each cell. In the following, we plot the NU-TF-MUSIC spectrum based on the elevation and azimuth angles of the proposed approach given by Error! Reference source not found.) for eight sources, where four interfering sources have the same elevation angle values as the four intended sources.

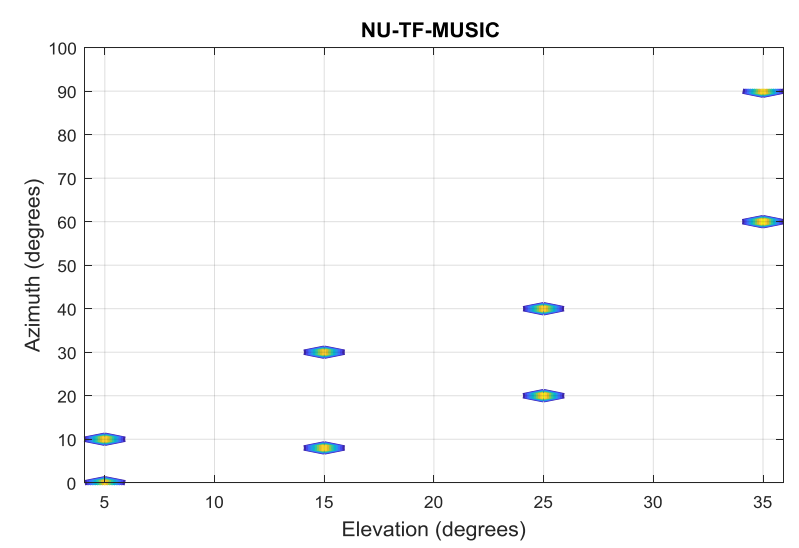

Figure 5. NU-TF-MUSIC spectrum for a SNR value of $15 \mathrm{~dB}$

Figure 5 depicts the angles estimation performance of the proposed algorithm with the $16 \times 8$ array configuration, a coherence interval of $C=128$, and a value of SNR $=15 \mathrm{~dB}$. The results show that elevation and azimuth angles can be clearly observed. The spectrum has been computed for all values of $\theta$ ranging from $0^{\circ}$ to $90^{\circ}$ and $\phi$ ranging from $-90^{\circ}$ to $90^{\circ}$, with an angular step size of $1{ }^{\circ}$. NU-TF-MUSIC spectrum exhibits a peak at each source location $\left(5^{\circ}, 0^{\circ}\right),\left(5^{\circ}, 10^{\circ}\right),\left(15^{\circ}, 8^{\circ}\right),\left(15^{\circ}, 30^{\circ}\right),\left(25^{\circ}, 20^{\circ}\right)$, $\left(25^{\circ}, 40^{\circ}\right),\left(35^{\circ}, 90^{\circ}\right)$ and $\left(35^{\circ}, 60^{\circ}\right)$.

Afterward, we present the RMSE of the proposed approach versus different SNR values and we compare the result for two types of the array configuration. 


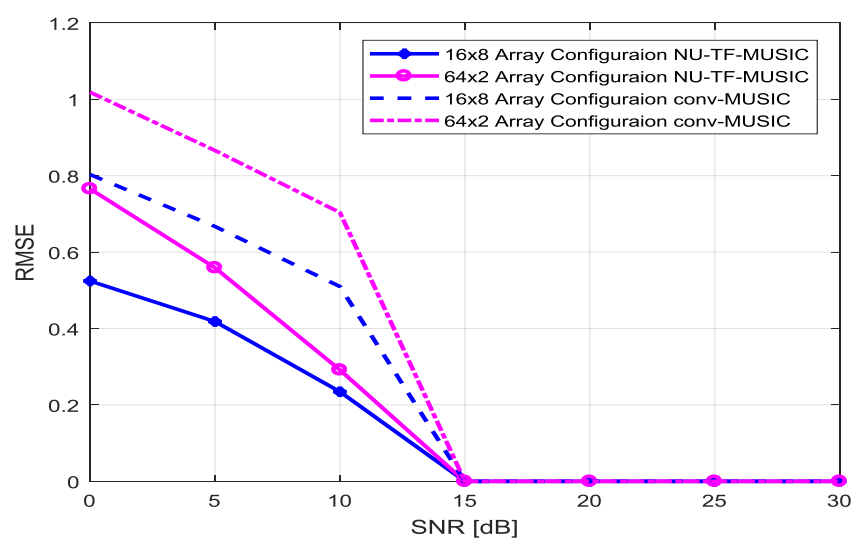

Figure 6. Comparison of RMSE of NU-TF-MUSIC and Conv-MUSIC versus SNR for different types of array configurations

We show in Figure 6, the comparison of RMSE of NonUnitary Time Frequency MUSIC method, denoted by NU-TF-MUSIC, and Conventional MUSIC method, denoted by Conv-MUSIC, versus different SNR values for the four interfering sources having the same elevation angle values as the four intended sources. The value of RMSE of both methods and for different types of arrays is under 1 with SNR ranges from $0 \mathrm{~dB}$ to $30 \mathrm{~dB}$. As it has been pointed out as the SNR increases, the performance of the two methods ameliorates. The performance of each array configuration for the two methods is illustrated by a curve as mentioned in Figure 6 . The $16 \times 8$ array configuration has acted in excellent quality for both NU-TF-MUSIC and Conv-MUSIC. Comparing the two array configurations, it is interesting to notice that the number of antenna element along $\mathrm{X}$ and/or $\mathrm{Y}$ axes contributes to improving the accuracy of angles estimation. The reason for which we observe a considerable difference between the RMSE values ranging from 0 to $15 \mathrm{~dB}$ for the two configurations. This open issue will be discussed with researchers of our Laboratory working on Antenna subjects.

Next, we display also the performance of the proposed method with another array geometry to see the impact of antenna geometry on our method. We choose the Uniform Circular Array UCA represented by the steering vector at any direction as in [31], [32]

$$
a_{c}(\theta, \phi)=\left[\begin{array}{c}
e^{j \frac{N}{2} \sin (\theta) \cos \left(\phi-\frac{2 \pi}{N}\right)} \\
e^{j \frac{N}{2} \sin (\theta) \cos \left(\phi-\frac{4 \pi}{N}\right)} \\
\vdots \\
e^{j \frac{N}{2} \sin (\theta) \cos (\phi-2 \pi)}
\end{array}\right]
$$

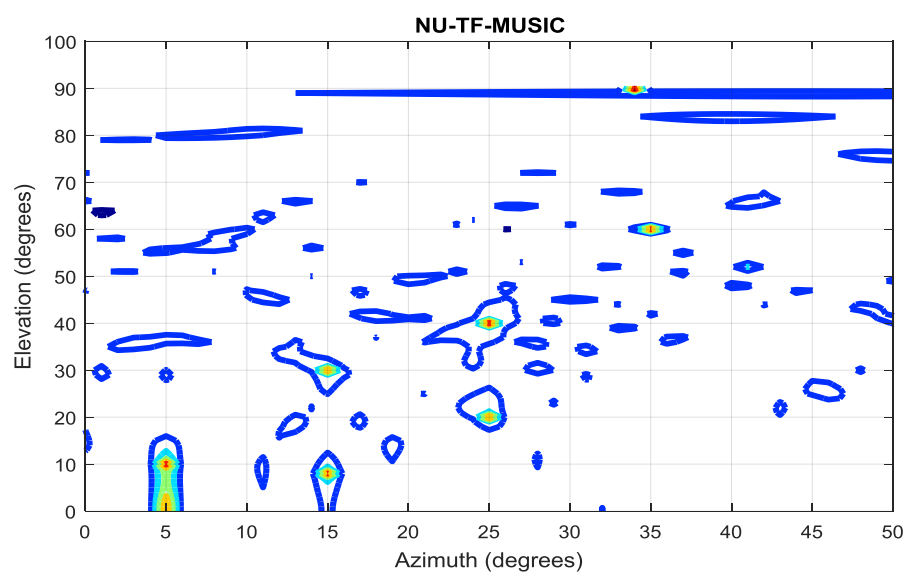

Figure 7. NU-TF-MUSIC spectrum for a UCA configuration and for a SNR value of $15 \mathrm{~dB}$ 
Figure 7 illustrates the angles estimation performance of the proposed algorithm for a UCA configuration, a coherence interval of $C=128$, and a value of $S N R=15 d B$. The elevation and azimuth angles can be clearly observed also for UCA configuration. We can conclude that our proposed method performs well for the URA and UCA configurations.

In the following, we choose the $16 \times 8$ array configuration to compute the rest of simulations. We evaluate the RMSE of NU-TF-MUSIC and Conv-MUSIC versus different SNR values for two number of samples values.

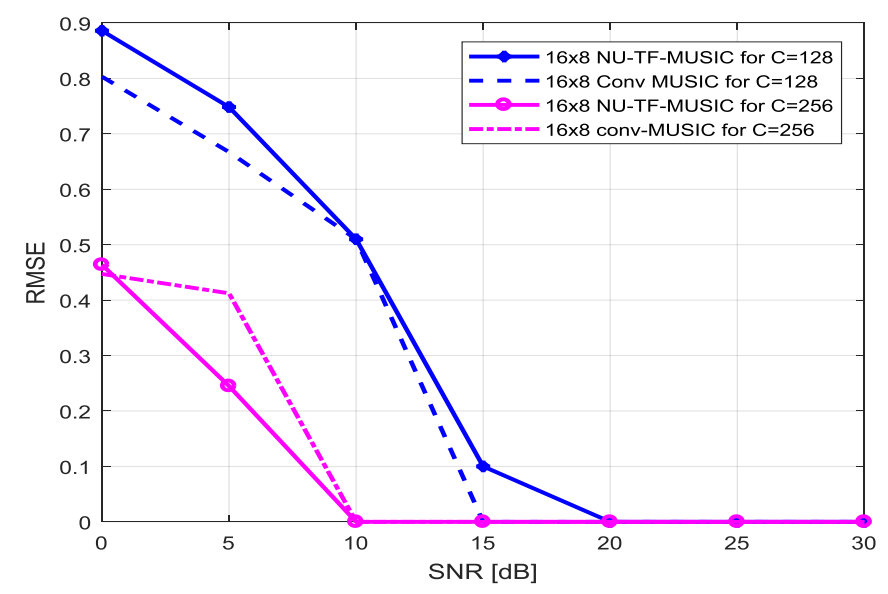

Figure 8. Comparison of RMSE of NU-TF-MUSIC and Conv-MUSIC versus SNR for different values of coherence time $\mathrm{C}$

Figure 8 presents the NU-TF-MUSIC and Conv-MUSIC with an array configuration of $16 \times 8$, and two different values of coherence interval C. Obviously, as we get more samples to estimate the steering matrix A, the angles estimation performance will enhance. All values of RMSE of the two methods are under 0.9 with SNR ranges from $0 \mathrm{~dB}$ to $30 \mathrm{~dB}$. The NU-TF-MUSIC method performs better than the Conv-MUSIC method for the two values of coherence interval $C=128$ and 256 .

To get a more quantitative understanding of how El-Az estimation method performs in a multi-Cell massive MIMO system, we display another simulation to prove what a massive antenna number can bring an enhancement in our approach performances.

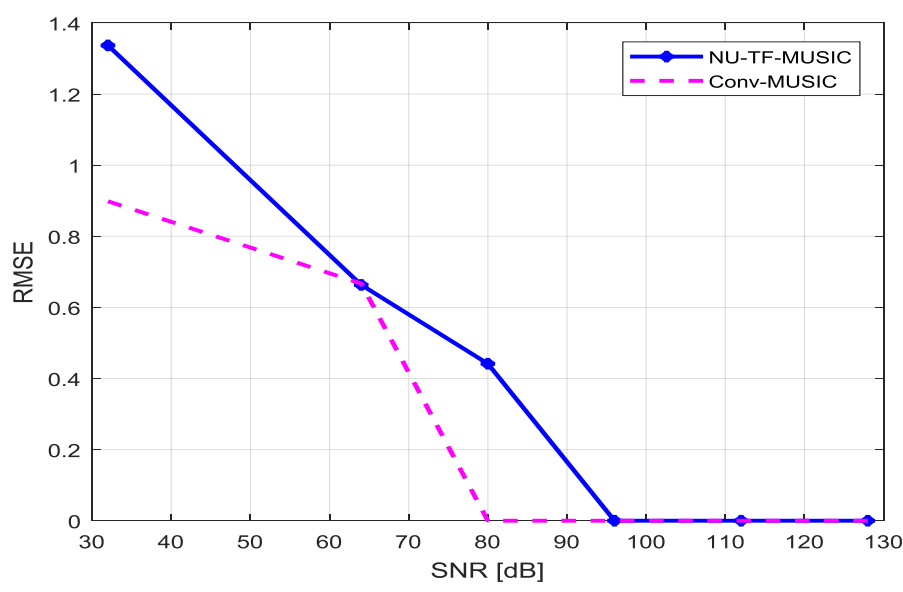

Figure 9. Comparison of RMSE of NU-TF-MUSIC and Conv-MUSIC versus the number of antenna elements for a $\mathrm{SNR}=15 \mathrm{~dB}$ and for four intended sources and four interfering ones 
We exhibit in Figure 9 the performance of the estimation error of the two methods versus the number of antenna elements $(32,64,80,96,112$ and 128) covering the eight sources for a SNR value of $15 \mathrm{~dB}$. The values of RMSE is under 1.4 for the antenna elements number of 32, 64, 80 and 96 for both methods. We can emphasize that as the number of antenna elements increases (beyond 96), the performance of NU-TF-MUSIC and Conv-MUSIC get more efficient. The analysis and simulations in Figure 9 note that the Conv-MUSIC method outperforms our solution. However, to get ameliorated performance, it is necessary to raise the number of realisations of our method to attain a good match between experimental and theoretical results. In our case, it was difficult due to the limitation of our computing equipment.

This study is a contribution to the ongoing discussions about the problem of pilot contamination outcoming from the reuse of pilots over cells in multi-cell Massive MIMO systems. We have addressed the problem of discriminating the target cell users and interfering ones especially those situated on edge of the cell. The originality of our solution lies in the fact that the pilot contamination is resolved as a source separation problem using time, frequency, and space variables. These results are in good agreement with the assumptions considered in the beginning stipulating that including 2D DOA (El and Az angles) would separate the users of each cell in contrast of other studies which considered a 1D DOA. One question still unanswered is whether this algorithm can be readily used in practice for real system with high dimensions.

\section{CONCLUSION}

In this paper, the pilot contamination problem, regarded as a bottleneck of Multi-Cell Massive MIMO Systems, was posed and solved as a sources separation problem over time, frequency and space domains. We have shown that including another parameter for DOA estimation (both elevation and azimuth) instead of 1D DOA (elevation only) brought more precision and succeed to discriminate users and enhance the sources separation in Multi-Cell Massive MIMO systems context. The idea fully exploits the concept of Spatial Time Frequency representations and their application to direction finding. We have introduced a new NU-TF-MUSIC method for El-Az estimation to distinguish between intended users and interfering ones. The result is an improved signal localization and separation using time, frequency, and space variables. Numerical experiments illustrated the accuracy and efficiency of the proposed technique for the Multi-Cell Massive MIMO systems.

\section{REFERENCES}

[1] N. Marchetti, "Towards the 5th Generation of Wireless Communication Systems", CoRR, vol. abs/1702.0, 2017.

[2] J. G. Andrews et al., "What Will 5G Be?", Sel. Areas Commun. IEEE J., vol. 32, no. 6, pp. 1065-1082, 2014.

[3] F. Boccardi, R. Heath, A. Lozano, T. L. Marzetta, and P. Popovski, "Five Disruptive Technology Directions for 5G”, IEEE Commun. Mag., vol. 52, no. 2, pp. 74-80, 2014.

[4] A. Rghioui and A. Oumnad, "Internet of Things: Surveys for measuring human activities from everywhere", Int. J. Electr. Comput. Eng., vol. 7, no. 5, p. 2474, 2017.

[5] D.-T. Do and D.-A. Nguyen, "The Maximal SINR Selection Mode for 5G Millimeter-Wave MIMO: Model Systems and Analysis", Indones. J. Electr. Eng. Comput. Sci., vol. 7, no. 1, pp. 150-157, 2017.

[6] S. Shinjo, K. Nakatani, K. Tsutsumi, and H. Nakamizo, "Integrating the Front End: A Highly Integrated RF Front End for High-SHF Wide-Band Massive MIMO in 5G", IEEE Microw. Mag., vol. 18, no. 5, pp. 31-40, 2017.

[7] G. Fodor et al., "An Overview of Massive MIMO Technology Components in METIS", IEEE Commun. Mag., vol. 55 , no. 6 , pp. $155-161,2017$.

[8] D. Gesbert, M. Kountouris, R. W. Heath, C. B. Chae, and T. Sälzer, "Shifting the MIMO Paradigm”, IEEE Signal Process. Mag., vol. 24, no. 5, pp. 36-46, 2007.

[9] G. Caire and S.Shamai, "On the Achievable Throughput of a Multi-Antenna Gaussian Broadcast Channel", IEEE Trans .Inf. Theory, vol. 49, no. 7, pp. 1691-1706.

[10] P. Viswanath and D. N. C. Tse, "Sum capacity of the vector Gaussian broadcast channel and uplink-downlink duality", IEEE Trans. Inf. Theory, vol. 49, no. 8, pp. 1912-1921, 2003.

[11] S. Vishwanath, N. Jindal, and A. Goldsmith, "Duality, Achievable Rates, and Sum-rate Capacity of Gaussian MIMO Broadcast Channels", IEEE Trans. Inf. Theory, vol. 49, no. 10, pp. 2658-2668, 2003.

[12] T. L. Marzetta, "How much training is required for multiuser MIMO?", Conf. Rec. - Asilomar Conf. Signals, Syst. Comput., pp. 359-363, 2006.

[13] F. Rusek et al., "Scaling up MIMO : Opportunities and Challenges with very large arrays", IEEE Signal Process. Mag., vol. 30, no. 1, pp. 40-60, 2013.

[14] A. Salh, L. Audah, N. S. M. Shah, and S. A. Hamzah, "Adaptive Antenna Selection and Power Allocation in Downlink Massive MIMO Systems”, Int. J. Electr. Comput. Eng., vol. 7, no. 6, pp. 3521-3528, 2017.

[15] T. L. Marzetta, "Noncooperative Cellular Wireless with Unlimited Numbers of base station Antennas", IEEE Trans. Wirel. Commun., vol. 9, no. 11, pp. 3590-3600, 2010.

[16] J. Nam, J. Y. Ahn, A. Adhikary, and G. Caire, "Joint Spatial Division and Multiplexing: Realizing massive MIMO gains with limited channel state information”, 2012 46th Annu. Conf. Inf. Sci. Syst. CISS 2012, vol. 1, no. 1, 2012. 
[17] E. Larsson, O. Edfors, F. Tufvesson, and T. Marzetta, "Massive MIMO for next generation Wireless Systems", IEEE Commun. Mag., vol. 52, no. 2, pp. 186-195, 2014.

[18] T. L. Marzetta, E. G. Larsson, B. Laboratories, and M. Hill, "Analysis of the Pilot Contamination Effect in very large Multicell Multiuser Mimo Systems for Physical Channel Models Hien Quoc Ngo Department of Electrical Engineering (ISY), Link o", no. 2, pp. 3464-3467, 2011.

[19] B. Gopalakrishnan and N. Jindal, "An Analysis of Pilot Contamination on multi-user MIMO Cellular Systems with many Antennas", IEEE Work. Signal Process. Adv. Wirel. Commun. SPAWC, pp. 381-385, 2011.

[20] T. M. F. Fernandez, A. Ashikhmin, "Interference Reduction on Cellular Networks with large Antenna Arrays", in Proc. of IEEE International Conference on Communications (ICC).

[21] L. M. Systems, H. Yin, D. G. Fellow, M. Filippou, and Y. Liu, "A Coordinated Approach to Channel Estimation", in IEEE J. Sel. Areas Commun., vol. 31, no. 2, pp. 264-273, 2013.

[22] R. R. Müller, L. Cottatellucci, and M. Vehkaperä, "Blind Pilot Decontamination", IEEE J. Sel. Top. Signal Process., vol. 8, no. 5, pp. 773-786, 2014.

[23] H. Yin, L. Cottatellucci, D. Gesbert, R. R. Müller, and G. He, "Robust Pilot Decontamination Based on Joint Angle and Power Domain Discrimination", pp. 1-14, 2015.

[24] A. Belouchrani and M. G. Amin, "Blind Source Separation based on Time-frequency Signal Representations", IEEE Trans. Signal Process., vol. 46, no. 11, pp. 2888-2897, 1998.

[25] Y. Zhang, W. Mu, and M. G. Amin, "Subspace Analysis of Spatial Time-frequency distribution Matrices", IEEE Trans. Signal Process., vol. 49, no. 4, pp. 747-759, 2001.

[26] L. Cohen, Time-frequency analysis, Prentice H. 1995.

[27] T. Wang, B. O. Ai, R. He, and Z. Zhong, "Two-Dimension Direction-of-Arrival Estimation for Massive MIMO Systems", IEEE Access, vol. 3, pp. 2122-2128, 2015.

[28] X. Cheng, C. Wang, and H. Wang, "Cooperative MIMO Channel Modeling and Multi-link Spatial Correlation Properties", Sel. Areas ..., 2012.

[29] K. Zheng, S. Ou, and X. Yin, "Massive MIMO Channel Models: A survey", Int. J. Antennas Propag., vol. 2014, 2014.

[30] A. Osseiran et al., "Scenarios for the 5G Mobile and Wireless Communications: the Vision of the METIS Project".

[31] Y. A. M. Dessouky, H. Sharshar, "Efficient Sidelobe Reduction Technique for small-sized Concentric Circular Arrays", Prog. Electromagn. Res., no. PIER 65, pp. 187-200, 2006.

[32] M. A. Ihedrane, S. Bri, and others, "High Resolution Method using Patch Circular Array", Int. J. Electr. Comput. Eng., vol. 7, no. 4, pp. 2116-2124, 2017. 